\title{
Latest results of the LHCf experiment
}

\author{
E. Berti ${ }^{a b}{ }^{a}$ O. Adriani, ${ }^{a b}$ L. Bonechi, ${ }^{a}$ M. Bongi, ${ }^{a b}$ R. D'Alessandro, ${ }^{a b}$ G. Castellini, \\ M. Haguenauer, ${ }^{d}$ Y. Itow, ${ }^{e f}$ K. Kasahara, ${ }^{g}$ Y. Matsubara, ${ }^{e}$ H. Menjo,,${ }^{e}$ Y. Muraki, ${ }^{e}$ \\ K. Ohashi, ${ }^{e}$ P. Papini, ${ }^{a}$ S. Ricciarini, ${ }^{a c}$ T. Sako, ${ }^{h}$ N. Sakurai, ${ }^{i}$ K. Sato,${ }^{e}$ Y. Shimizu,,${ }^{j}$ \\ L. Silveri, ${ }^{a b}$ T. Tamura, ${ }^{k}$ A. Tiberio,${ }^{a b}$ S. Torii, ${ }^{k}$ A. Tricomi, ${ }^{l m}$ W. C. Turner,${ }^{n}$ \\ M. Ueno, ${ }^{e}$ K. Yoshida ${ }^{g}$ \\ ${ }^{a}$ INFN Section of Florence - Florence, Italy \\ ${ }^{b}$ University of Florence - Florence, Italy \\ ${ }^{c}$ IFAC-CNR - Florence, Italy \\ ${ }^{d}$ Ecole-Polytechnique - Palaiseau, France \\ ${ }^{e}$ Institute for Space-Earth Environmental Research - Furo-cho, Chikusa-ku, Nagoya, Japan \\ ${ }^{f}$ Kobayashi-Maskawa Institute for the Origin of Particles and the Universe, Nagoya University - \\ Nagoya, Japan \\ ${ }^{g}$ Faculty of System Engineering, Shibaura Institute of Technology, Japan \\ ${ }^{h}$ Institute for Cosmic Ray Research, University of Tokyo, Chiba, Japan \\ ${ }^{i}$ Tokushima University, Tokushima, Japan \\ ${ }^{j}$ Kanagawa University - Kanagawa, Japan \\ ${ }^{k}$ RISE, Waseda University - Shinjuku, Tokyo, Japan \\ ${ }^{l}$ INFN Section of Catania - Catania, Italy \\ ${ }^{m}$ University of Catania - Catania, Italy \\ ${ }^{n}$ LBNL - Berkeley, California, USA \\ E-mail: eugenio.berti@fi.infn.it
}

The LHCf experiment is designed to provide precise measurements of the production spectra relative to neutral particles produced in the very forward region by high energy proton-proton and proton-ion collisions. This information is necessary in order to test and tune hadronic interaction models used by ground-based cosmic rays experiments. In order to reach this goal, LHCf makes use of two small sampling calorimeters installed in the LHC tunnel at $\pm 140 \mathrm{~m}$ from IP1, both able to detect neutral particles having pseudo-rapidity $\eta>8$.4. In LHC Run II, LHCf acquired data relative to $\mathrm{p}-\mathrm{p}$ collisions at $\sqrt{s}=13 \mathrm{TeV}$ and $\mathrm{p}-\mathrm{Pb}$ collisions at $\sqrt{s_{N N}}=8.1 \mathrm{TeV}$. In this paper, we discuss the results obtained from $\mathrm{p}$-p collisions at $\sqrt{s}=13 \mathrm{TeV}$, focusing in particular on photon and neutron production, the two analyses already published by the collaboration.

PACS: $13.85 .-t, 13.85 . \mathrm{Tp}$

European Physical Society Conference on High Energy Physics - EPS-HEP2019 -

10-17 July, 2019

Ghent, Belgium

${ }^{*}$ Speaker. 


\section{Introduction}

The measurement of the flux and composition of cosmic rays up to the Greisen-ZatsepinKuzmin (GZK) cut-off is necessary to understand the mechanisms responsible for their acceleration and propagation in the universe. These quantities are measured by ground-based experiments through the indirect detection of the extensive air showers (EASs) that cosmic rays form when interacting with the atmosphere. The properties of the primary particle are then reconstructed making use of Monte Carlo simulations that, being EASs physics described by soft QCD, necessarily rely on phenomenological models. The variation among them is very significant at high energies, resulting in large systematic uncertainties on cosmic rays measurements. The main aim of the LHC forward (LHCf [1]) experiment is to give important information for the calibration of hadronic interaction models in the phase space relevant for EASs physics. This is obtained measuring the distribution of neutral particles produced in the very forward region by high energy proton-proton and proton-ion collisions. The Large Hadron Collider (LHC [2]) at CERN is the most suitable place where to perform these measurements, because a center of mass energy of $\sqrt{s}=13 \mathrm{TeV}$ in p-p collisions is equivalent to an energy of about $9 \times 10^{16} \mathrm{eV}$ in the reference frame where the target is at rest, a value not so smaller than the one of Ultra High Energy Cosmic Rays (UHECRs).

\section{The experiment}

The LHCf experiment consists of two small sampling calorimeters installed in the LHC tunnel at $\pm 140 \mathrm{~m}$ from IP1, the interaction point dedicated to ATLAS [3]. Being placed beyond the D1 dipole magnet, only neutral particles produced in p-p and p-ion collisions and having pseudorapidity $\eta>8.4$ can reach the experiment. Each one of the two detectors, called Arm1 and Arm2, is made up by two square towers of $22 \mathrm{~W}$ and $16 \mathrm{GSO}$ layers for a total length of $22 \mathrm{~cm}$, equivalent to $44 X_{0}$ and $1.6 \lambda_{I}$. Towers sizes are $20 \mathrm{~mm} \times 20 \mathrm{~mm}$ and $40 \mathrm{~mm} \times 40 \mathrm{~mm}$ for Arm1, $25 \mathrm{~mm}$ $\times 25 \mathrm{~mm}$ and $32 \mathrm{~mm} \times 32 \mathrm{~mm}$ for Arm2. Energy resolution is better than 5\% for $\gamma_{\mathrm{s}}$ above 100 $\mathrm{GeV}$ and about $40 \%$ for hadrons above $500 \mathrm{GeV}$. The transverse position of the incident particle is reconstructed using $4 \mathrm{X} / \mathrm{Y}$ imaging layers inserted at different depths. They are formed by $1 \mathrm{~mm}$ width GSO-bars in the case of Arm1 and by $160 \mu \mathrm{m}$ read-out pitch silicon microstrip detectors in the case of Arm2. Position resolution is better than $200 \mu \mathrm{m}$ for $\gamma \mathrm{s}$ above $100 \mathrm{GeV}$ and $1 \mathrm{~mm}$ for hadrons above $500 \mathrm{GeV}$. More detailed descriptions of the detector are reported elsewhere $[4,5]$.

\section{Analysis results}

The data relative to p-p collisions at $\sqrt{s}=13 \mathrm{TeV}$ was acquired by the LHCf experiment during Fill 3855, a low luminosity and high $\beta^{*}$ fill. Using this data set, the collaboration published two analyses relative to photons [6] and neutrons [7] production in the forward region.

The differential neutron production cross section was measured using only the data from the Arm 2 detector. Fig. 1 shows the measured distributions after energy unfolding, which is mandatory given the limited resolution. As we can see, the limitations of models are clearly evident for $\eta>$ 10.76, where none of them is able to reproduce the observed peak structure at around $5 \mathrm{TeV}$. In the other regions they have better but not satisfactory perfomances, with SIBYLL 2.3 [8] and EPOSLHC [9] having the best overall agreement with data for $8.99<\eta<9.22$ and $8.81<\eta<8.99$, 

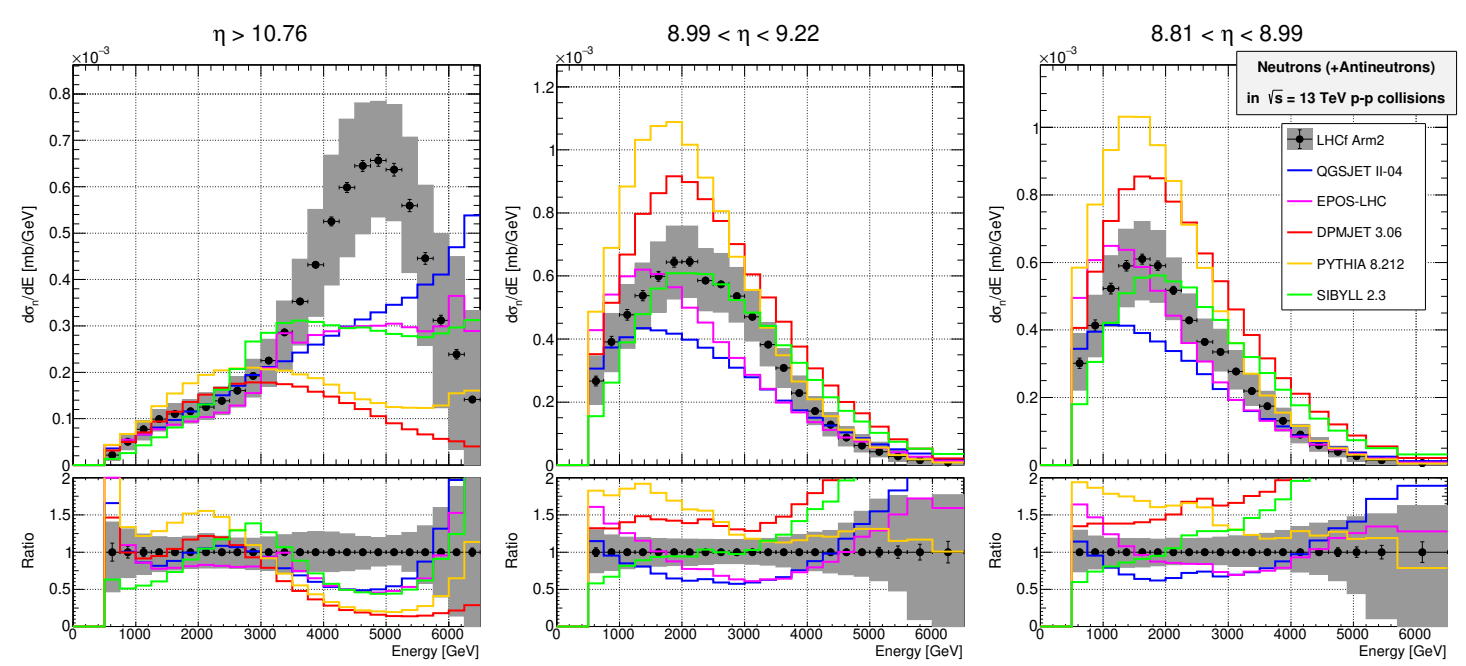

Figure 1: Differential neutron production cross section for p-p collisions at $\sqrt{s}=13 \mathrm{TeV}$, measured using the Arm2 detector and compared with model predictions [7]. Black markers represent the experimental data with statistical errors, whereas gray bands represent the quadratic sum of statistical and systematic uncertainties. Colored histograms refer to models predictions at the generator level.

respectively. The collaboration is currently working to the extension of the analysis to other regions of the Arm1 and Arm2 detectors in order to enlarge the pseudorapidity coverage. In this way, we can have enough measurements to derive fundamental quantities relative to EAS development, like the forward production cross section, the differential energy flow and the average inelasticity.

The differential photon production cross section was measured combining the data of Arm1 and Arm 2 detectors. In addition, a more detailed study is ongoing exploiting the fact that the LHCf and ATLAS experiments had common operations in LHC Run II. In this way, it is possible not only to measure the inclusive photon production in the forward region, but also to distinguish between the diffractive and non-diffractive contributions. According to Monte Carlo studies [10], diffractive events can be identified with a purity of almost $100 \%$ and an efficiency of about $50 \%$ by requiring a central veto, defined as zero charged tracks with $\mathrm{p}_{\mathrm{T}}>100 \mathrm{MeV}$ in $|\eta|<2.5$. Using this simple selection, the two experiments released a conference note on the role of diffraction in the production of forward photons [11]. Fig.2 top shows the experimental measurements compared to generators distributions, both with and without central veto condition, whereas Fig. 2 bottom shows the ratio between the distributions without and with central veto condition. The ratio of the distributions is especially significant for our study, both because some uncertainties cancel out leading to smaller error bands and because it directly tests the relative impact of diffraction mechanism. Here we can note two important points. The first one is that in both cases no model is able to reproduce satisfactorily the data in all the energy range. Among all generators considered here, EPOS-LHC is the one having the best overall agreement. The second one is that the ability of a model to reproduce the data is different with or without central veto. For example, in $\eta>10.94$, QGSJET II-04 [12] is slightly softer than data without central veto, but with central veto is strongly softer at low energy. 

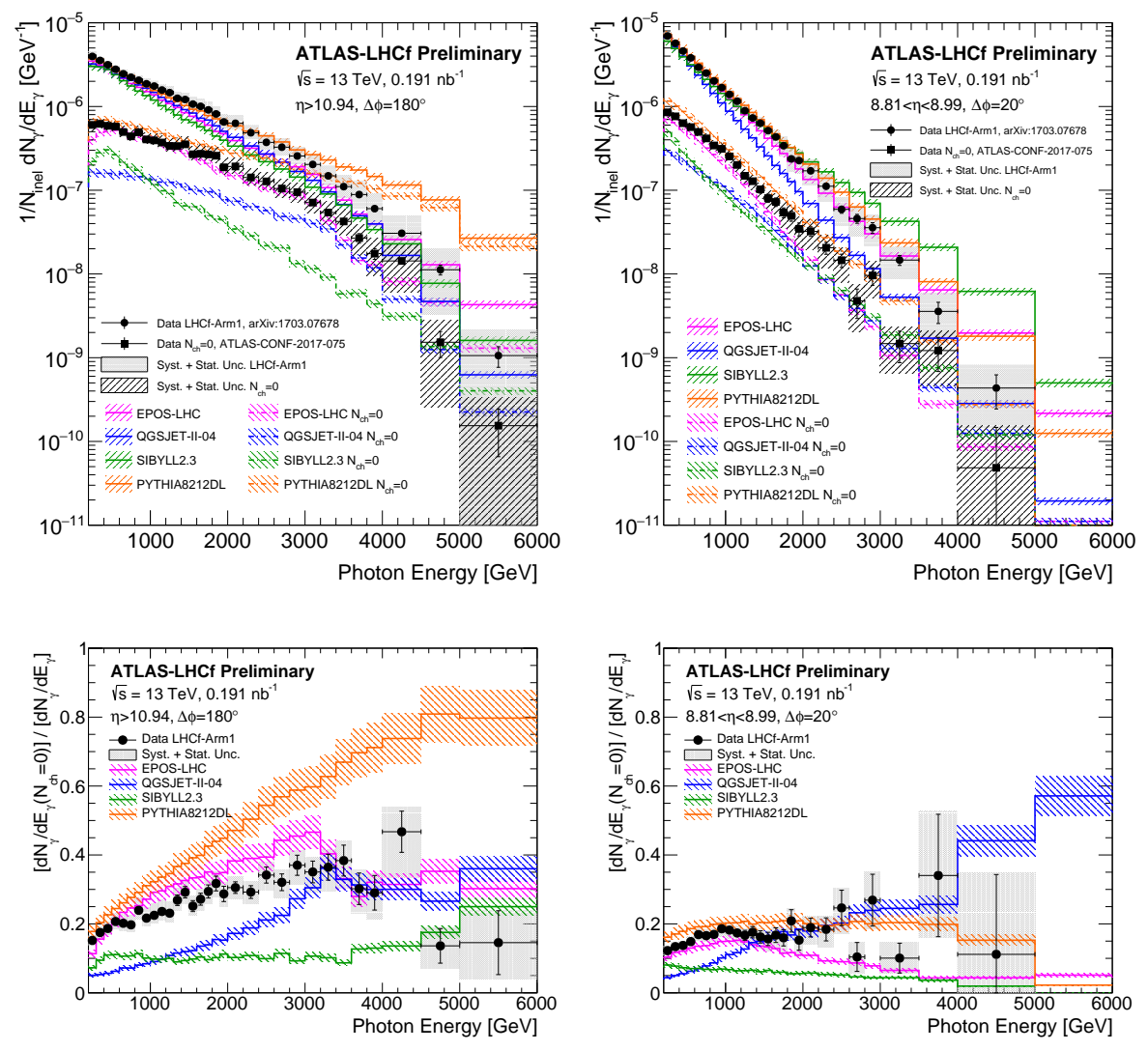

Figure 2: Preliminary photon energy spectra relative to $\sqrt{s}=13 \mathrm{TeV}$ p-p collisions, measured using the Arm1 detector and compared with model predictions [11]. Top shows the distributions with and without central veto condition (square and circle markers for data, dashed and solid line for generators), whereas bottom shows the ratio between the distributions without and with $\mathrm{N}_{\mathrm{ch}}=0$ condition. Black markers represent the experimental data with statistical errors, whereas gray bands represent the quadratic sum of statistical and systematic uncertainties. Colored histograms refer to models predictions at the generator level.

This with/without central veto discrepancy is expected for all models because diffractive and non diffractive mechanisms are treated independently inside them. Thus, it is crucial to separately measure the role of the two contributions to forward particle production and this is the reason of the large activity dedicated to this analysis, which, after its extension to the Arm2 detector, will be published soon. Afterwards, a similar approach will be applied to the neutron case, where, as discussed in a recent study [13], it is expected to have a significant impact thanks to its implication in the study of central/forward production asymmetry.

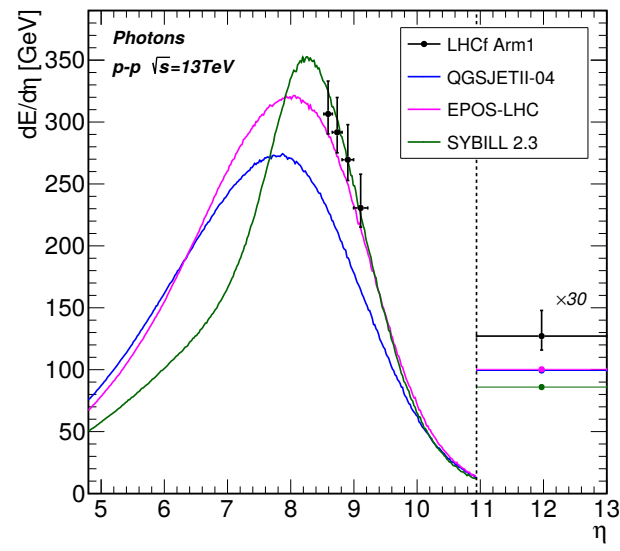

Figure 3: Preliminary photon energy flow relative to $\sqrt{s}=13 \mathrm{TeV}$ p-p collisions [14]. Black markers are the values measured using the Arm1 detector together with the quadratic sum of statistical and systematic uncertainties. Colored lines refer to model predictions from QGSJET II-04, EPOS-LHC, SIBYLL 2.3. 
Another interesting result is the measurement of the photon differential energy flow, which can be obtained using numerical integration on the inclusive spectra shown in Fig.2. In addition, thanks to the large acceptance of the Arm1 detector in the region $\eta<9.22$, it was possible to extend the analysis adding three points at smaller pseudorapidities. This is a very important point because it allows us to measure the energy flow near the region where it is expected to be maximum, which is, according to most models, around $\eta \sim 8$. Fig. 3 shows the preliminary photon energy flow [14], where we can see that, for $\eta>10.94$, no model agrees with data, whereas, for $8.52<\eta<9.22$, SIBYLL 2.3 is in good agreement with the measurements, EPOS-LHC is slightly softer but still compatible, while QGSJET II-04 leads to a significant underestimation.

\section{Conclusions and future prospects}

In LHC Run II, LHCf acquired data relative to p-p collisions at $\sqrt{s}=13 \mathrm{TeV}$ and $\mathrm{p}-\mathrm{Pb}$ collisions at $\sqrt{s_{N N}}=8.1 \mathrm{TeV}$. Regarding data relative to $\mathrm{p}$-p collisions at $\sqrt{s}=13 \mathrm{TeV}$, photon and neutron inclusive results are published, while $\pi^{0}$ analysis is still ongoing. In parallel, exploiting the powerful LHCf-ATLAS joint analysis, an accurate measurement of diffractive and non-diffractive contributions to forward production is almost completed for photons and now starting for neutrons. All the results obtained so far showed that in the forward region no model agrees well with the experimental observations. Recently, the LHC research board approved the LHCf operation plan in LHC Run III, involving two different dedicated data takings: p-p collisions at $\sqrt{s}=14 \mathrm{TeV}$ and p-O collisions at $\sqrt{s_{N N}}=9.9 \mathrm{TeV}$. The first one is required to decrease the statistical uncertainty on $\pi^{0}$ measurements, exploiting a new trigger scheme and an increased DAQ rate. The second one is fundamental to study forward particle production in a scenario that is very similar to the first interaction of a high energy cosmic ray with an atmospheric nucleus.

\section{References}

[1] O. Adriani et al., Journal of Instrumentation 3 (2008) S08006.

[2] L. Evans, New Journal of Physics 9 (2007) 335.

[3] G. Aad et al., Journal of Instrumentation 3 (2008) S08003.

[4] T. Mase et al., Nuclear Instruments and Methods in Physics Section A A671 (2012) 29-136.

[5] K. Kawade et al., Journal of Instrumentation 9 (2014) P03016.

[6] O. Adriani et al., Physics Letters B 780 (2018): 233-239.

[7] O. Adriani et al., Journal of High Energy Physics (2018) 2018: 73.

[8] F. Riehn et al., PoS ICRC 2015 (2016) 558.

[9] T. Pierog et al., Physical Review C 92 (2015) 034906.

[10] Q. D. Zhou et al., European Physical Journal C 77 (2017) 212.

[11] The ATLAS and LHCf collaborations, CERN Preprint (2017) ATLAS-CONF-2017-075.

[12] S. Ostapchenko, Physical Review D 83 (2011) 014018.

[13] S. Ostapchenko et al., Physical Review D 94 (2016) 114026.

[14] Y. Makino et al., CERN Thesis (2017) CERN-THESIS-2017-049. 